\title{
Los artistas que trabajan para el Rey: La Junta de Obras y Bosques
}

\author{
María Victoria García Morales *
}

La casa de Austria organizó sus Estado como una inmensa herencia particular que debe mantener y engrandecer. El Rey es quién ostenta el poder y el control de todos los asuntos. Una organización administrativa perfectamente diseñada desde los Reyes Católicos, le informa y exige su dictamen.

La acción del Estado se realiza a través de los diversos Consejos que asumen una serie de competencias, delegadas del Rey, para su gobierno. Como descargo al abundamiento de funciones en estos Consejos surgen las Juntas, con idéntica misión de gobierno y autonomía.

Los Reyes Austrias, por educación y por deseo grandes clientes del Arte, han ido acumulando una serie de bienes muebles e inmuebles que necesitan unas directrices de gobierno para su conservación. La creación de la Junta de Obras y Bosques, en tiempo de Carlos $V$, responde al deseo de la Corona por velar y cuidar de sus posesiones, de su patrimonio particular, reflejo de su poder.

En el origen de su fundación, esta Junta asume unas reponsabilidades que ocupaban al Rey, pero en el transcurso del tiempo su libertad de acción, competencias, eficacia de su gestión, variarán. Al iniciarse el siglo XVII, después de gobernar España un monarca tan férreamente disciplinado como Felipe II, quien asume en su persona todos los quehaceres, la Junta de Obras y Bosques pierde actividad, sin apenas asuntos que le competan, hasta el extremo de que el Duque de Lerma, valido del

- Articulo presentado, con ligeras modificaciones, en el VII Congreso Español de Historia del Arte, Murcia, 1988. 
Rey Felipe III, tiene intención de extinguir esta Junta, despachando él, tras la información del Secretario, los asuntos directamente con el Rey.

Un memorial del Secretario de la Junta dirigido en el año de 1610 al Duque de Lerma', refleja, al relatar su historia, la situación de este organismo en el cambio de centuria: en primer lugar se refiere a un tiempo en que una única persona resuelve con el Rey Felipe II todos los despachos: compras de dehesas, heredamientos, etc.. y que posteriormente el Rey Felipe III nombra una serie de personas para que conformen la Junta y gobierne su Patrimonio pero sin plena autonomía, a pesar de la relevancia de los componentes (miembros del Consejo o de la Cámara), pues «...siempre se le comunicaba a él lo que se ofrecia...":

"... más como los negocios que tenian dificultad ya eran pocos tambien las juntas se tenian muy raras veces porque todo lo resolvia Su Majestad, que haya gloria, por lo que yo (el Secretario, quien escribe el memorial) le consultaba y después de su muerte sabe Vuestra Excelencia (el Duque de Lerma, a quien escribe) la instancia que hicieron el Marqués de Poza y el licenciado Ramirez de Prado para que se volviere a fundar esta Junta y concurriesen en ella ellos..."

y otros varios que enumera, entre los que es de señalar la presencia del Confesor Real, su pertenencia a esta Junta es constante en todo el siglo. "... sucediendo los unos confesores a los otros...".

$\mathrm{Y}$ el documento sigue contando cómo:

“... muerto el Marqués de Poza, cesó la Junta y Vuestra Excelencia me escribió que se podia escusar dando yo cuenta de todo a Vuestra Excelencia para que dándole Vuestra Excelencia a su Majestad se resolviese y ejecutase todo con más brevedad y aunque corrió asi muchos meses, el Conde de Villalonga y Ramirez de Prado comenzaron a tener esta Junta sin mi intervención y a cabo de muchos meses procuraron que el Presidente de Indias - y otros varios-entrasen en ella y después mandó su Majestad que yo concurriese con ellos...".

A partir de este momento que se puede situar en 1606 (por la coincidencia de nombre citados en este memorial, con los enumerados como

AGP. Sección Administrativa. Legajo 853. Año 1610. 
pertenecientes a la Junta en la Cédula de 19 de junio de $1606^{2}$ y a pesar del deseo manifestado en contra del Duque de Lerma, los nombramientos para formar parte de la Junta se suceden:

“... eligiendo las personas que su Majestad era servido en lugar de los que faltaban...",

así como los decretos reales por los que se amplian sus competencias.

La Junta de Obras y Bosques durante este siglo XVII vive una edad de oro, cuenta con el apoyo real, un amplio marco de ejercicio y sus miembros pertenecen a la minoría más selecta, mas su eficacia real es limitada: sus dictámenes no siempre prevalecieron, la tramitación de los asuntos es lenta, las quejas por las demoras en la resolución de los asuntos y pleitos son frecuentes (aunque el tiempo ha de tener otro valor) "...ha mas de 7 años...» y por el hecho principal de que la Corona en esta centuria carece de medios económicos para aumentar sus posesiones o emprender grandes programas constructivos y un nuevo palacio como el Buen Retiro, en un principio se exime de su jurisdicción por lo que ha de limitarse a un programa artístico de mantenimiento.

Durante todo el siglo (aunque con ligeras variaciones: frecuencia de las sesiones, número de asistentes, recursos financieros, que influyen en su gestión), la máquina administrativa de la Junta ejerció sus funciones con celo, estando presente e informando constantemente, si bien acompasada al ritmo mortecino de una administración que se ahoga a sí misma. Por ello, al llegar su supresión, está abierta con antelación, la reforma iniciada desde que los Borbones ocupan el trono, ha ido distrayendo sus funciones a otros organismos, buscando cauces más rápidos, - los primeros Borbones habian asumido en su persona el gobierno de los Sitios Reales próximos a la Corte y encargado la dirección a los Secretarios de Estado-, aunque a esta Junta le seguian compitiendo los asuntos judiciales.

\section{MISIONES DE LA JUNTA DE OBRAS Y BOSQUES}

Podemos conocer los objetivos que el Emperador Carlos señaló a esta Junta, si empezamos por el final, es decir, a partir de la Cédula

2 AGP. Cedulas Reales. Registro 10, folio $541 \mathrm{v}$. 
emitida en 1768, por la que se suprime definitivamente esta Real Junta al carecer de contenido ${ }^{3}$.

El decreto de extinción, marca claramente, la voluntad real de su fundación:

“... Sabed que la Junta de Obras y Bosques y todas sus oficinas fueron creadas por los Reyes mis gloriosos Progenitores, para el régimen, gobierno y cuidado de los Palacios. Alcázares y Bosques Reales: de la fábrica de edificios nuevos: de las Obras y reparos que se ofrecian en ellos y sus Jardines, y de la conservación de la Caza de sus Bosques y Cotos con privativa jurisdición en las materias de Justicia y Gobierno...".

Asimismo, a esta Junta le compete la consulta para la provisión de los Oficios en estas demarcaciones. Oficios que comprenden tanto el personal de dirección, administrativo y justicia, como artistas que trabajan para el Rey. Por lo que hemos decidido nuestro estudio en mostrar la organización de este instituto, pues de él depende el programa artístico de la Corte, y su actuación en el caso concreto de la selección de maestros que han de trabajar para la Corona.

Así pues a la Junta de Obras y Bosques, responsable del crecimiento y mantenimiento de las propiedades del Rey, se le conceden las prerogativas necesarias para ejercer su función, que comprende no sólo los asuntos de gobierno y aprovechamiento de la Hacienda del Rey, sino también la administración de justicia en su jurisdición. Se puede decir que es un organismo autónomo, que no depende de ninguna otra instancia más que del Rey, es su representante por lo que uno de sus principales objetivos será cumplir la voluntad real ${ }^{4}$, aunque tampoco duda defender sus facultades ante él.

Si bien las competencias de la Junta están fijadas desde su fundación, su presencia en ciertos momentos se oscurece ${ }^{5}$, llegando incluso a discutirse su supresión, pero (como ya hemos dicho) con el nuevo siglo

3 AHN. Sección Consejos. Legajo 49526.

${ }^{4}$ A este deseo corresponde el origen del empleo de Superintendente, que la Junta otorga al marqués de Malpica porque "...como el Marqués asistía de ordinario a Palacio. podría entender la real voluntad de Vuestra Majestad cerca de las obras que fuese servido se hiciera...".

s Cuando el Rey Felipe II despacha directamente los asuntos de obras, cuando el Duque de Lerma intenta hacerse con sus facultades. 
la Junta adquirirá nuevo vigor, quizá a instancia de los nobles recelosos del poder del valido.

Tarea principal de la Junta desde este momento, para potenciar su desarrollo, es estar informada de todo lo referente a sus competencias, para ello logra del Rey este permiso por lo que es normal en los encabezamientos de sus órdenes la fórmula "... porque el Rey desea conocer..." utilizando su dependencia real como parapeto de su eficacia.

Al iniciarse el siglo, las Instrucciones o leyes para el gobierno de los diferentes Sitios, están dadas, pero reiteradamente desoidas. Ahora la Junta, para ejercer sus competencias y velar por la buena administración de las posesiones del Rey, "exige su cumplimiento", para ello concede comisiones y ordena visitas e inspecciones a los diversos lugares para, según los resultados presentados por los comisionados, actuar con toda autoridad, bien interviniendo con el consejo para mejorar el funcionamiento o castigando las infracciones encontradas. Le compete la labor de redactar estas leyes, Instrucciones, así las del gobierno general de las Casas, como las dictadas para el desarrollo de las obras, a este respecto es de señalar la Instrucción de 1605 para las obras del Alcázar de Madrid, que responde al deseo de poner orden, ante el incumplimiento de lo establecido y observado en la inspección realizada ${ }^{6}$.

Como el éxito de una gestión depende mucho de ejercer el control de la asignación económica, intenta organizar la hacienda, para conocer la realidad de su haber y recursos que tiene para administrar, por ello las órdenes a Alcaides o Gobernadores, para realizar inventario de sus bienes muebles e inmuebles, se suceden ?

En este proceso, de querer asumir sus responsabilidades, adquiere "... porque el Rey quiere brevedad...", el privilegio de despachar Cédulas y libranzas, sin que el Consejo de Hacienda y la Contaduria Mayor las refrende y emita, simplemente han de librarlas y anotar en sus libros ${ }^{8}$. Algo más adelante se nombra, para que tenga cuenta y razón de todo 10 que hay en las Casas Reales, Alcázares y Heredamientos al primer Contador de Obras y Bosques ${ }^{9}$, subordinado al Secretario, porque «... El Rey quiere que haya entera noticia en la Junta de mis Obras y Bosques,

${ }^{6}$ Esta Instrucción será modelo para las obras que se inicien en la centuria.

A este respecto las actas de la Junta de estos primeros años del siglo estan llenas de información (Libro 2301 de la Junta de Obras y Bosques Reales).

${ }^{8}$ AGP. Cédulas Reales. Registro 10, folio $541 \mathrm{v}$.

${ }^{9}$ AGP. Sección Administrativo Legajo 853 año 1612. 
donde por mi mandado se trata de la conservación y gobierno de las dichas Casa Reales...".

La Junta, informada, dueña de sus recursos, establecida las leyes, no duda en intervenir y cumplir sus competencias, sin dejar que ningún otro organismo o persona le arrebate el más mínimo poder ${ }^{10}$. El factor económico, elemento fundamental en la historia de la Junta, y la reponsabilidad de sus miembros, son la causa de su agotamiento ${ }^{11}$.

Sin dinero pocos asuntos de los que la competen puede resolver: las sesiones, al carecer de contenido, se distancian y limitan a cumplir trámites burocráticos, por lo que la inasistencia se hace frecuente, con lo que ella misma abandona su misión antes de decidirse su supresión.

Mas anulada la Junta y repartidas sus competencias, su organización y sistema de control será un modelo para la organización borbónica, como se desprende del decreto de extinción ${ }^{12}$.

\section{LA DIRECCIÓN DE LA JUNTA DE OBRAS Y BOSQUES}

Para cumplir estas misiones la Junta contó con una dirección compuesta por personas allegadas al Rey, nobles de alto prestigio y una trama administrativa con diversas oficinas, servidas por diferente clases de dependientes.

En el escrito de 1610, ya mencionado, del Secretario de la Junta de Obras y Bosques al Duque de Lerma, observamos que los cargos que ocupan las personas citadas son importantes: Presidente de órdenes, Contador, miembros del Consejo y Cámara, Presidente de Hacienda, ofi-

10 A este respecto podemos citar la figura del Superintendente que en determinadas ocasiones intenta abrogarse competencias que no tenia.

"Senalábamos la coincidencia de fechas en que se reclama la asistencia a las reuniones de los pertenecientes, con memoriales de la Junta en que solicita sus consignaciones porque su hacienda tiene quiebra (Archivo General de Simancas. Casas y Sitios Reales. Legajo 309, folio 66 y siguientes y AGP. Seccion Administrativa. Legajo 853.)

12 El primer Secretario de Estado manejará los asuntos económicos y gubernativos de los Palacios y Bosques del Rey, según los antecedentes buscados en sus archivos, por lo que se exige la entrega, bajo inventario formal, de los Papeles y Libros que custodia la Secretaria de la Junta. Todas las cuentas de Sitios Reales, que controlaba la Contaduria de la Razón General de Obras y Bosques, se remitirán, a traves del primer Secretario de Estado. a la Contaduria Mayor de Cuentas. Los asuntos judiciales y contenciosos pasaran a la Sala de Justicia del Consejo. que actuará “... del mismo modo y bajo las mismas reglas que lo hacia la Junta...", por lo que todos los procesos existentes en la Escribania de Camara de la Junta, han de pasar al archivo del Consejo. 
cios que por si no implican pertenecer a la Junta, forman parte de ella en virtud de sus personas: procedentes de la nobleza ${ }^{13}$, y en el mismo documento leemos más adelante:

"... y no he visto que se haya atendido tanto a los oficios como a las personas en la elección de ellas...".

“... ni que sea anexo al presidente de Hacienda presidir ni concurrir a esta Junta, aunque tengo por conveniente -es el parecer del Secretario- que se halle en ella...".

No obstante, asi no debe permanecer durante toda la centuria, y con el correr del tiempo habrá cargos que llevarán implícito el pertenecer a la Junta: en un despacho al Duque del Infantado, en $1612^{14}$, se dice:

"... que entre en la dicha Junta el Duque del Infantado, como Mayordomo Mayor...".

A fin de siglo, en 1695, se puede afirmar que son muchos los puestos que conllevan pertenecer a la Junta. Asi un oficio, particular, dirigido al Secretario de la Junta ${ }^{15}$, se pide información sobre “... Todas las personas que tienen voto en la Junta de Obras y Bosques regularmente según su institución..." y seguidamente se incluye la relación ${ }^{16}$, y continúa citando los nombres de los que en ese momento conforman la Junta. La lista de miembros de la Junta es mayor que la de los empleos que dan derecho a pertenecer a ella, ésto hace pensar que el Rey sigue nombrando por merced particular.

La dignidad es necesaria para pertenecer a la Junta como se desprende del memorial que remite la Junta de Obras y Bosques al Rey, en respuesta a un despacho real por el que se consulta si Juan Bautista

${ }_{13}$ El Confesor del Rey es el único no perteneciente a este estamento.

if AGP. Seccion Administrativo. Legajo 853. Año 1612.

AGP. Sección Administrativo. Legajo 853. Año 1695.

16 «... El Presidente de Castilla, el Mayordomo Mayor del Rey Nuestro Señor, el Cazador Mayor, el Montero Mayor, el Presidente de Hacienda. el padre Contesor de S.M., el Alcaide del Buen Retiro, el Alcaide del Pardo, el Ministro más antiguo, de la Cámara de Castilla. otro Ministerio del mismo Consejo que tambien ha sido el más antiguo en algunas ocasiones, también tiene entrada el Superintendente de las obras, cuando le hay...". 
Crescencio podría ser elegido miembro de la Junta ${ }^{17}$. Destacamos el parecer en contra del Marqués de Alcañizas, que dice que es conforme a que se le conceda servir a S.M., pero no cree que sea oportuno para «... tan gran Tribunal...» porque:

"... constituyéndose de tan graves personas, que los más son primogénitos de su casa, y de las mayores de estos Reinos, no dice bien con el estado de Juan Bautista...".

Otra opinión singular emite el Conde de la Enseyra, no relacionada con su linaje, sino velando por la Hacienda Real, en la que se advierte cierto recelo hacia la persona extranjera.

A partir de 1620, los deseos del Rey por honrar a sus vasallos nombrándoles miembros de este Tribunal son frecuentes, mas la designación de tan crecido número no implica una reunión multitudinaria. Es frecuente que los nombrados excusen su asistencia a las sesiones periodicas, aludiendo a desplazamientos con el Rey, coincidencia con otro Consejo, principalmente debido a la acumulación de cargos o mercedes en unos pocos: el estamento noble de la sociedad. Estas ausencias no incomodan al Rey, más bien, al contar con ellas es por lo que sus nombramientos proliferan. En los acuerdos de las sesiones, el Secretario puntualiza:

$$
\begin{aligned}
& \text { "... se despachan los negocios con los que se pudieron hallar en } \\
& \text { ella...". }
\end{aligned}
$$

y en los márgenes, donde aparece la relación de asistentes, vemos que el número más frecuente es el de cinco, aunque también «... se podrá tener la Junta con los tres que quedan..." ${ }^{18}$.

La incomparecencia de los ministros designados a las tan frecuentes sesiones

"... Su Majestad tiene mandado que la Junta de Obras y Bosques se tenga dos días por semana..." ${ }^{19}$.

\footnotetext{
AGP. Sección Administrativo. Legajo 853. Año 1630

AGP. Sección Administrativo. Legajo 853. Año 1610

Ibidem
} 
y el incumplimiento de esta norma:

“... ha más de un mes que no se convoca la Junta de Obras y Bosques..." ${ }^{20}$.

restará eficacia a esta Junta, por cuanto no se despacharán los asuntos con la suficiente agilidad, contribuyendo con esta dejación a que otros organismos asuman sus competencias.

A principios del XVII, no era habitual el nombramiento formal para pertenecer a ella, simplemente bastaba la elección del Rey:

"... no he visto que se haya despachado Cédula ni otro recaudo para entrar en esta Junta..., ${ }^{21}$

y otro documento posterior, de $1621^{22}$, el Rey dice:

“... en la Junta de Obras y Bosques ha de entrar el Marqués de Malpica, gentilhombre de la Camara de los Reyes, mi señores Padre y Abuelo, y aunque no se haya despachado Célula de ello ordeneis que desde luego entre en ella...”.

Sin embargo, el nombramiento por Cedula Real, conforme avanza el tiempo se hace necesario, y encontramos muy poco posterior al documento aludido y referido asimismo al Marqués de Malpica, que el Rey dice a la Junta ${ }^{23}$.

“... despachareis Cédula y me la enviareis a firmar...”.

AGP. Sección Administrativo. Legajo 853. Año 1693.
AGP. Sección Administrativo. Legajo 853. Año 1610.
AGP. Sección Administrativo. Legajo 853. Año 1621.
AGP. Sección Administrativo. Legajo 853. Año 1621. 
Aún otro papel se ocupa del nombramiento de Malpica ${ }^{24}$ es en el que se le pregunta con qué título quiere que se le nombre en la Cédula, a lo que contesta:

"... junto a Marqués de Malpica, podrá V.m. poner en la Cédula. Mariscal de Castilla..."

que habla de su alta estima.

La fórmula establecida en el anterior nombramiento será la habitual: el Rey elige las personas que componen "Su" Ministerio, sin parecer alguno de los demás de la Junta, y ordena al Secretario:

"... vos lo direis en ella y siendo necesario Cédula para ello, se la despachareis en la forma que se acostumbra... ${ }^{25}$,.

"... vos lo direis en la Junta y si fuere menester despacharle alguna Cédula se hará en la forma que se suele..."

La Junta cumple el deseo del Rey, admite al nuevo designado, y emite la Cédula, que él firmará

"... La forma que se suele...", es una expresión que indica un mecanismo y una fórmula escrita establecidos por la rutina administrativa. La redacción de las Cédulas es pues muy similar en todos los nombramientos, que ejemplificamos en la Cédula para el nombramiento del Conde de Castro ${ }^{26}$. En un primer apartado el Rey expone la legitimidad de la Junta, la continuidad desde su establecimiento y demarcaciones Reales:

"... Por cuanto por mi mandado se va continuando la Junta que hasta aqui ha habido para el gobierno y administración de mis Alcazares Reales, Ingenio de la Moneda de Segovia. Heredamiento de Aranjuez.

${ }^{24}$ Abordando un tema "interesante" de este siglo: el protocolo, causa de competencias y litigios, segun las preeminencias que acompañan a cada titulo o nombramiento.

${ }^{25}$ Legajo 853. nombramientos del Presidente de Castilia y del Duque del Infantado, si bien este último, en este momento, no entrará por las competencias con el Presidente de Hacienda.

${ }^{2 t}$ AGP. Cédulas Reales Registro 13, folio 307. 
fábrica y patronato del Monasterio de San Lorenzo el Real y todo 10 demás tocante y concerniente a mis Obras y Bosques en la forma que se hacia en tiempo de los Reyes, mis señores abuelo y padre que hayan gloria...".

Otra parte está compuesta por la designación propiamente dicha: el Rey manifiesta la estima que profesa al elegido, se cita su nombre y méritos, y se hace alusión a la necesidad de su presencia por lo que se le concede el nombramiento que le faculta para despachar los asuntos, en igualdad a los demás miembros. Seguimos el documento anterior:

"... por la satisfacción que tengo de Vos D. Gomez Manriqueda Mendoza. Conde de Castro, mi Mayordomo Mayor y a lo que pueda importar vuestra asistencia para la buena dirección de las cosas y materias que se tratan en la dicha Junta, he tenido por bien entreis en ella y despacheis con los demás que alli concurren, todos los negocios tocantes al ministerio de mis Obras y Bosques..."

Señala a continuación las misiones que desarrolla la Junta y añade:

"... que para todo lo referido y cada cosa y parte de ello os doy tan cumplido poder como se requiere y es necesario y lo han tenido y tienen los demás que me han servido y sirven en la dicha Junta que asi es mi Voluntad...".

Por último, la fórmula legal de quien toma la razón, dónde se registra $y$ fecha.

\section{LA TRAMA ADMINISTRATIVA DE LA JUNTA DE OBRAS Y BOSQUES}

Para su buen gobierno se desarrolla una amplia trama administrativa que actúa al mandado de la dirección de la Junta de Obras y Bosques, elegida directamente por el Rey. 
La variedad de materias atendidas hace crear una serie de departamentos u oficinas que entienden de los diferentes asuntos: "Secretaria", resuelve los negocios económicos y gubernativos, dando cauce a las representaciones, instancias y recursos que se presentan a la oficina correspondiente; toma juramento y expide el título necesario para ejercer el trabajo para el que se ha sido seleccionado. "Agencia Fiscal. Contaduría de la Razón General», recibe, distribuye y justifica el presupuesto económico asignado a la Junta. "Escribanía de Cámara», a quien conciernen los asuntos judiciales.

Las personas que trabajan para la Junta pertenecen al servicio del Rey, a él compete su elección pues sólo ha delegado "la consulta para la provisión de los oficios", es decir, la Junta tiene facultad para convocar la vacante existente, recibir los memoriales de los interesados, seleccionar y proponer los candidatos que cree más convenientes, emitiendo su parecer sobre el mejor, pero será la Volutad Real quien finalmente decida, la más de las veces, según el criterio propuesto por su Junta.

El estar al servicio de la Corona es una merced o un honor que el Rey concede y muchos de sus súbditos desean, más por la mentalidad del momento que por los beneficios que comporta ${ }^{27}$, si bien en una época de crisis económica el empleo fijo es una seguridad.

Se denominaban Oficiales Reales (Mayores y Menores) a los que el Rey elegía y le servian en todas partes, firmaba Cédula de nombramiento, despachada por la Junta, y cobraban de la Hacienda de Casas Reales en virtud de sus nóminas. Otro tipo de personal es el de servicio u Ordinario, era nombrado por el Alcaide, Gobernador $u$ Oficiales del Sitio y cobraba, asimismo, de la Hacienda Real para Obras, y no se le despachaba título ${ }^{28}$.

Hemos encontrado varias órdenes por las que el Rey recaba información a la Junta acerca de empleos que provee, gratificaciones que concede, ayuda de costas, bienes muebles de sus casa, etc. que demuestran el constante interés real por estar informado de su Patrimonio, y la autonomía "controlada" de que goza la Junta.

Para el Rey austria, es una obsesión mantener y conservar la herencia recibida. Se puede leer este pensamiento en varios nombramientos para pertenecer a la Junta en que dice:

${ }^{27}$ Son innumerables las quejas de patronos y trabajadores por el escaso salario y la demora en recibirlo.

28 Ambas categorias de oficio, Oficiales Reales y Ordinarios, son de pie fijo, que significaba que el salario se paga de la Hacienda Real en virtud de nóminas. 
“... por la satisfacción que se ha de dar de las obras que se hicieron en su tiempo (Padre y Abuelo), como de los daños que hizo la caza...".

Por una de estas órdenes conocemos una relación de los Oficios Mayores y Menores que se ocupan en los distintos Sitios y oficinas, acompañados de su salario ${ }^{29}$. Consta en primer lugar, como es habitual, el Confesor del Rey (que goza del sueldo más elevado de todos los enumerados), luego el Montero y el Cazador Mayor; -para la Secretaria: el Secretario, el Oficial Mayor, el Portero, etc.; para las distintas oficinas: el Abogado fiscal, procurador, Contador, Alcalde Juez de Bosques, Escribano, Alguacil Fiscal y a continuación señala los Oficiales existentes en cada posesión y los Ordinarios, que nombran los Alcaides u Oficiales, cobran, sin título de Su Majestad, por salario o jornal.

El número y variedad de dependientes va en razón de la importancia del lugar, por ejemplo: el Alcázar de Madrid, la Casa de la Moneda de Segovia, el Heredamiento de Aranjuez, presentan una larga nómina, mientras que otros Sitios: Casa de Vaciamadrid, la Casa y Bosque de Balsain, la fábrica y bosques de San Lorenzo el Real, cuentan con un máximo de cuatro empleados.

El salario que se asigna a cada empleo depende, como es lógico, de su categoria, por lo que las nóminas más elevadas corresponden a: Veedores, Maestros Mayores, Pagadores, pero incluso la cuantia de éstas, varia en los diferentes Lugares, estando, por lo general, mejor pagado el Oficial de Madrid ${ }^{30}$. Se establece el salario en metálico, pero también puede complementarse con partidas anuales de trigo y cebada, como ocurre en: el Pardo, Heredamiento de Aranjuez y El Escorial; asimismo, constan los Oficios que tienen derecho a médico, botica y casa de aposento, son estas algunas de las "ventajas" que se citan, sin especificar, en los nombramientos, cuando se dice “... con las prerrogativas, preeminencias y honores que como a tal - se especifica el Oficiole corresponden...".

Cada posesión cuenta con el personal necesario para su gobierno y obras, pero por motivos principalmente económicos se mantiene el im-

${ }^{29}$ Es un documento fechado en 1625 que responde a la informacion solicitada desde el Consejo de Hacienda “... porque Su Majestad lo desea saber...". El documento presenta diversas tachaduras que hacen suponer un aprovechamiento posterior de esta relación: el Cardenal Duque, aparece tachado.

${ }^{30}$ Por alguna circunstancia especial, puede darse el caso que un Aparejador, por ejemplo el de Granada, reciba más salario que el de Madrid. 
prescindible, por ello es frecuente conceder la compatibilidad de cargos $^{31}$, o la suspensión de alguno, cuando ya no se considere oportuno $^{32}$, o recibir el empleo como honor, sin salario alguno ${ }^{33}$.

La Junta por su Secretaría interviene directamente en los asuntos de Obras y por lo tanto sus oficiales dependen directamente de ella. El gobierno y administración de los diferentes Sitios se realiza por los Alcaides y Gobernadores, unas veces nombrados a propuesta de la Junta, otras por la Cámara (cuando es perpétuo). Tanto Oficiales de Obras, como Alcaides y Gobernadores tienen la potestad para nombrar al personal subalterno ${ }^{34}$.

${ }^{31}$ Por ejemplo en Segovia, Valladolid, del Juzgado de Obras y Bosques se ocupan los Corregidores de la ciudad y no tiene salario de la Junta.

${ }_{32}$ En Granada, Toledo. Aranjuez, se deja consumir por falta de quehacer el puesto de Maestro de Obras.

${ }_{33}$ El Alcaide de! cuarto de San Jeronimo.

${ }^{34}$ Cuando el Alcaide es perpetuo, como Sevilla. Valladolid con el Duque de Lerma, mas tarde el Buen Retiro con el Conde Duque de Olivares, y poco a poco muchos mas, 10 que supuso un vacio de contenido en la Junta, tiene poder para nombrar a todos los oficiales. 